\title{
Periodic lattices in Minkowski space
}

\author{
A. D. Boozer ${ }^{\text {a) }}$ \\ Department of Physics, California Institute of Technology, Pasadena, California 91125
}

(Received 29 March 2010; accepted 26 July 2010)

\begin{abstract}
We describe a set of periodic lattices in (1+1)-dimensional Minkowski space, where each lattice has an associated symmetry group consisting of inhomogeneous Lorentz transformations that map the lattice onto itself. Our results show how ideas of crystal structure in Euclidean space generalize to Minkowski space and provide an example that illustrates basic concepts of spacetime symmetry. (C) 2010 American Association of Physics Teachers.

[DOI: $10.1119 / 1.3480023$ ]
\end{abstract}

\section{INTRODUCTION}

In Euclidean space one can describe various crystal structures in which repeated units are arranged in a periodic lattice. ${ }^{1,2}$ Such a crystal structure has an associated space group consisting of rigid, distance-preserving motions that map the crystal lattice onto itself. In this paper, we consider the analogous problem for Minkowski space. We describe a set of periodic lattices in $(1+1)$-dimensional spacetime, where each lattice has an associated group consisting of inhomogeneous Lorentz transformations that map the lattice onto itself. This group of transformations forms a discrete subgroup of the inhomogeneous Lorentz group. ${ }^{4-7}$ Our results show how basic ideas of crystal structure in Euclidean space generalize to Minkowski space. The problem of finding periodic lattices in $(1+1)$-dimensional Minkowski space was first considered in Ref. 3, but this work is couched in the formal language of mathematical crystallography and is likely to be inaccessible to many readers.

We can compare our periodic lattices in $(1+1)$ dimensions with two previous schemes for discretizing spacetime in $(3$ +1 ) dimensions. Ordinarily, spacetime is taken to be a continuum, in which the spacetime coordinates $x^{\mu}=(t, x, y, z)$ are real numbers. In one scheme, due to Schild, ${ }^{8}$ continuum spacetime is replaced with a cubic lattice in which the coordinates $x^{\mu}$ are integer multiples of a fixed lattice constant $L$. In another scheme, due to Coish, the real number system is replaced with a finite field, and the coordinates $x^{\mu}$ are taken to be elements of that field. ${ }^{9,10}$ For both these schemes, one first specifies a spacetime lattice and then determines its symmetry properties.

In this paper, we approach the problem in the reverse direction: we require that our spacetime lattices be invariant under a discrete subgroup of the Lorentz group and then determine the lattices that meet this requirement. The paper should be accessible to undergraduates who have had some exposure to special relativity, and could be used to supplement a classroom discussion of spacetime symmetry. The paper could also be used to supplement an undergraduate class in condensed matter physics because it shows how ideas of crystal structure can be applied in a novel context.

In Sec. II we briefly review the inhomogeneous Lorentz group for $(1+1)$-dimensional Minkowski space. In Sec. III we describe the problem of finding a spacetime lattice that is invariant under a discrete subgroup of the inhomogeneous Lorentz group. In Sec. IV we give a general solution to this problem. In Secs. V and VI we describe several example lattices. In Sec. VII we show how our results can be generalized to higher dimensions.

\section{THE INHOMOGENEOUS LORENTZ GROUP}

Let us consider Minkowski space in $(1+1)$ dimensions. We will label events in Minkowski space using coordinates $x^{\mu}=(t, x)$, where $x^{0}=t$ is the time at which the event occurs and $x^{1}=x$ is its spatial location. Given a pair of events $x_{1}^{\mu}$ and $x_{2}^{\mu}$, we define the spacetime interval $\Delta s^{2}$ between the two events by

$$
\Delta s^{2}=\eta_{\mu \nu}\left(x_{1}^{\mu}-x_{2}^{\mu}\right)\left(x_{1}^{\nu}-x_{2}^{\nu}\right),
$$

where

$$
\eta_{\mu \nu}=\left(\begin{array}{cc}
1 & 0 \\
0 & -1
\end{array}\right)
$$

is the Minkowski metric tensor.

Let us now consider two types of coordinate transformations from the original coordinates $x^{\mu}$ to new coordinates $\bar{x}^{\mu}$. One type of transformation corresponds to a boost by a velocity $\beta$. We will denote this type of transformation by $B(\beta)$ :

$$
\bar{x}^{\mu}=B(\beta) x^{\mu}=\Lambda_{\nu}^{\mu}(\beta) x^{\nu},
$$

where

$$
\Lambda_{\nu}^{\mu}(\beta)=\left(\begin{array}{cc}
\gamma & \beta \gamma \\
\beta \gamma & \gamma
\end{array}\right)
$$

is the Lorentz transformation matrix and $\gamma \equiv\left(1-\beta^{2}\right)^{-1 / 2}$. A second type of transformation corresponds to a translation through a fixed displacement $d^{\mu}$. We will denote this type of transformation by $T\left(d^{\mu}\right)$ :

$$
\bar{x}^{\mu}=T\left(d^{\mu}\right) x^{\mu}=x^{\mu}+d^{\mu} .
$$

By using Eqs. (1), (3), and (5), it is straightforward to show that both types of transformations leave the spacetime interval $\Delta s^{2}$ invariant:

$$
\Delta s^{2}=\eta_{\mu \nu}\left(\bar{x}_{1}^{\mu}-\bar{x}_{2}^{\mu}\right)\left(\bar{x}_{1}^{\nu}-\bar{x}_{2}^{\nu}\right) .
$$

Let us now consider a general coordinate transformation that consists of an arbitrary sequence of boosts and translations. One can show that such a transformation is equivalent to a single boost $B(\beta)$ followed by a single translation $T\left(d^{\mu}\right)$, for some values of the parameters $\beta$ and $d^{\mu}$. We will denote this general type of transformation by $G\left(d^{\mu}, \beta\right)$ :

$$
\bar{x}^{\mu}=G\left(d^{\mu}, \beta\right) x^{\mu}=T\left(d^{\mu}\right) B(\beta) x^{\mu}=\Lambda_{\nu}^{\mu}(\beta) x^{\nu}+d^{\mu} .
$$

Because boosts and translations both leave the spacetime interval $\Delta s^{2}$ invariant, so does a general coordinate transformation $G\left(d^{\mu}, \beta\right)$. It is straightforward to check that the set of all 
such general coordinate transformations forms a group, where the group product is taken to be the composition of transformations. This group is known as the inhomogeneous Lorentz group. ${ }^{11}$

\section{SPACETIME LATTICES}

In analogy with crystal structure in ordinary Euclidean space, we would like to find a periodic lattice in Minkowski space that is invariant under a discrete subgroup $H$ of the inhomogeneous Lorentz group. That is, for any event $x^{\mu}$ in the lattice and any transformation $G\left(d^{\mu}, \beta\right)$ in the group $H$, the event $G\left(d^{\mu}, \beta\right) x^{\mu}$ should also belong to the lattice. We will assume that the lattice consists of events of the form

$$
R_{m, n}^{\mu}=m e_{1}^{\mu}+n e_{2}^{\mu},
$$

where $m$ and $n$ are integers, and $e_{1}^{\mu}$ and $e_{2}^{\mu}$ are basis vectors. $^{12}$

Let us now consider the symmetry properties of the lattice. The lattice is invariant under translation by an arbitrary lattice vector $R_{m, n}^{\mu}$. We will denote such a translation by $T_{m, n}$ :

$$
T_{m, n} x^{\mu} \equiv T\left(R_{m, n}^{\mu}\right) x^{\mu}=x^{\mu}+R_{m, n}^{\mu} .
$$

We would also like the lattice to be invariant under boosts of certain specific velocities. Suppose the lattice is invariant under a boost of velocity $\beta>0$. We will denote such a boost by $B_{1}$ :

$$
B_{1} x^{\mu} \equiv B(\beta) x^{\mu}=\Lambda_{\nu}^{\mu}(\beta) x^{\nu} .
$$

By symmetry, the lattice must also be invariant under a boost of the opposite velocity, which we will denote by $B_{-1}$ :

$$
B_{-1} x^{\mu} \equiv B(-\beta) x^{\mu}=\Lambda_{\nu}^{\mu}(-\beta) x^{\nu} .
$$

For an arbitrary nonzero integer $r$, we define $B_{r}$ to be a sequence of $r$ boosts $B_{1}$ if $r>0$ and a sequence of $|r|$ boosts $B_{-1}$ if $r<0$ :

$$
B_{r} \equiv\left\{\begin{array}{cl}
\left(B_{1}\right)^{r} & \text { if } r>0 \\
\left(B_{-1}\right)^{|r|} & \text { if } r<0 .
\end{array}\right.
$$

One can show that $B_{r}=B\left(\beta_{r}\right)$, where

$$
\beta_{r}=\frac{(1+\beta)^{r}-(1-\beta)^{r}}{(1+\beta)^{r}+(1-\beta)^{r}}
$$

Because the lattice is invariant under boosts $B_{1}$ and $B_{-1}$, it is also invariant under the sequence of boosts $B_{r}$. There is a distinct boost $B_{r}$ for every nonzero integer $r$, so there are an infinite number of boosts that leave the lattice invariant. In contrast, for a crystal lattice in Euclidean space there are only a finite number of rotations that leave the lattice invariant.

We now consider a general transformation of the lattice that consists of an arbitrary sequence of boosts and translations. As before, one can show that such a transformation is equivalent to a single boost $B_{r}$ followed by a single translation $T_{m, n}$, and we will denote such a transformation by $G_{m, n, r}=T_{m, n} B_{r}$. The set of all such transformations forms a group $H$ that is a discrete subgroup of the inhomogeneous Lorentz group.

What remains to be done is to determine the allowed velocities $\beta$ such that the boost $B(\beta)$ leaves the lattice invari- ant. For $B(\beta)$ to leave the lattice invariant, it is necessary and sufficient that $B(\beta) e_{1}^{\mu}$ and $B(\beta) e_{2}^{\mu}$ belong to the lattice. ${ }^{13}$ Thus, we require that

$$
\begin{aligned}
& \Lambda_{\nu}^{\mu}(\beta) e_{1}^{\nu}=a e_{1}^{\mu}+b e_{2}^{\mu}, \\
& \Lambda_{\nu}^{\mu}(\beta) e_{1}^{\nu}=c e_{1}^{\mu}+d e_{2}^{\mu}
\end{aligned}
$$

for some set of integers $\{a, b, c, d\}$. Our goal is to determine the possible basis vectors $e_{1}^{\mu}$ and $e_{2}^{\mu}$ and sets of parameters $\{\beta, a, b, c, d\}$ for which Eq. (13) holds. It turns out that it is more convenient to work in terms of $\gamma$ rather than $\beta$, and thus in what follows we will take the lattice parameters to be $\{\gamma, a, b, c, d\}$ and define $\beta \equiv\left(1-1 / \gamma^{2}\right)^{1 / 2}$.

\section{SOLUTION TO THE PROBLEM}

We proceed as follows. It is useful to define new basis vectors $e_{+}^{\mu}$ and $e_{-}^{\mu}$ by

$$
e_{+}^{\mu}=(1,1), \quad e_{-}^{\mu}=(1,-1) .
$$

From Eq. (4) for $\Lambda_{\nu}^{\mu}(\beta)$, it follows that

$$
\Lambda_{\nu}^{\mu}(\beta) e_{+}^{\nu}=\lambda e_{+}^{\nu}, \quad \Lambda^{\mu}{ }_{\nu}(\beta) e_{-}^{\nu}=\lambda^{-1} e_{-}^{\nu},
$$

where $\lambda \equiv \gamma(1+\beta)$. Thus, the basis vectors $e_{+}^{\mu}$ and $e_{-}^{\mu}$ are eigenvectors of $\Lambda^{\mu}{ }_{\nu}(\beta)$ with eigenvalues $\lambda$ and $1 / \lambda$, respectively. The solution for $\gamma$ in terms of $\lambda$ is

$$
\gamma=(1 / 2)(\lambda+1 / \lambda) \text {. }
$$

We can express the original basis vectors $e_{1}^{\mu}$ and $e_{2}^{\mu}$ in terms of the new basis vectors $e_{+}^{\mu}$ and $e_{-}^{\mu}$ :

$$
e_{1}^{\mu}=A e_{+}^{\mu}+B e_{-}^{\mu}, \quad e_{2}^{\mu}=C e_{+}^{\mu}+D e_{-}^{\mu}
$$

for some values of $A, B, C$, and $D$. From Eqs. (13), (16), and (18), it follows that

$$
\begin{array}{ll}
\lambda A=a A+b C, & \lambda^{-1} B=a B+b D, \\
\lambda C=c A+d C, & \lambda^{-1} D=c B+d D .
\end{array}
$$

We can express Eqs. (19) and (20) in the form

$$
\lambda \vec{v}_{+}=M \vec{v}_{+}, \quad \lambda^{-1} \vec{v}_{-}=M \vec{v}_{-},
$$

where

$$
\vec{v}_{+}=\left(\begin{array}{l}
A \\
C
\end{array}\right), \quad \vec{v}_{-}=\left(\begin{array}{l}
B \\
D
\end{array}\right), \quad M=\left(\begin{array}{ll}
a & b \\
c & d
\end{array}\right) .
$$

We see that $\vec{v}_{+}$and $\vec{v}_{-}$are eigenvectors of $M$ with eigenvalues $\lambda$ and $1 / \lambda$, respectively. The trace of a matrix is equal to the sum of its eigenvalues, and hence

$$
a+d=\lambda+1 / \lambda=2 \gamma,
$$

where we have used Eq. (17) for $\gamma$. The determinant of a matrix is equal to the product of its eigenvalues, and hence

$$
a d-b c=1 \text {. }
$$

Because $\gamma \geq 1$, Eq. (23) implies that $a+d \geq 2$. The conditions $a+d \geq 2$ and $a d-b c=1$ are necessary and sufficient for obtaining a viable spacetime lattice. Once we have specified the integers $\{a, b, c, d\}$ that satisfy these conditions, the corresponding value of $\gamma$ is given by Eq. (23). Because $a$ and $d$ are integers, Eq. (23) implies that $\gamma$ must be either an integer or a half-integer. From Eqs. (23) and (24), it follows that if 


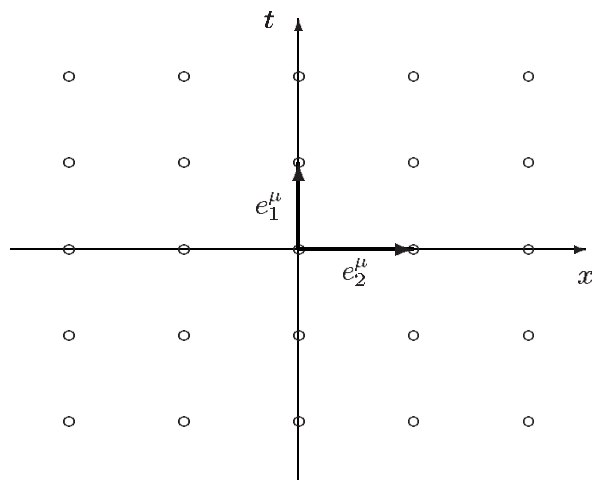

Fig. 1. Spacetime lattice generated by the basis vectors $e_{1}^{\mu}$ and $e_{2}^{\mu}$ defined in Eq. (28). The circles indicate events $R_{m, n}^{\mu}$ that belong to the lattice.

$b=0$ or $c=0$, then $a=d=1$, so $\gamma=1$ and $\Lambda^{\mu}{ }_{\nu}(\beta)$ is the identity transformation. Because this solution is trivial, in what follows we will always assume that $b \neq 0$ and $c \neq 0$.

We have determined the allowed lattice parameters $\{\gamma, a, b, c, d\}$, but have yet to determine the allowed basis vectors $e_{1}^{\mu}$ and $e_{2}^{\mu}$. From Eq. (19), it follows that the components of $e_{1}^{\mu}$ and $e_{2}^{\mu}$ must satisfy

$$
C / A=(\lambda-a) / b, \quad D / B=\left(\lambda^{-1}-a\right) / b .
$$

The four components $\{A, B, C, D\}$ correspond to the four degrees of freedom in choosing the basis vectors $e_{1}^{\mu}$ and $e_{2}^{\mu}$. Equation (25) fixes two of these degrees of freedom, leaving two degrees of freedom remaining. One degree of freedom corresponds to the freedom to scale the entire lattice by arbitrary scale factor $\eta$ :

$$
\{A, B, C, D\} \rightarrow\{\eta A, \eta B, \eta C, \eta D\} .
$$

A second degree of freedom corresponds to the freedom to boost the entire lattice by an arbitrary gamma factor $\gamma$ $=(1 / 2)(\eta+1 / \eta)$ :

$$
\{A, B, C, D\} \rightarrow\{\eta A, B / \eta, \eta C, D / \eta\} .
$$

Note that if the components $\{A, B, C, D\}$ satisfy Eq. (25), then so do the scaled and boosted components described by Eqs. (26) and (27).

\section{RECTANGULAR LATTICES}

We now consider a special class of lattices whose basis vectors $e_{1}^{\mu}$ and $e_{2}^{\mu}$ have the form

$$
e_{1}^{\mu}=(T, 0), \quad e_{2}^{\mu}=(0, L)
$$

for some constants $T$ and $L$. A lattice of this form is shown in Fig. 1.

To clarify the physical meaning of a spacetime lattice of this form, let us consider a specific set of events that constitute such a lattice. Imagine that we construct a series of flashing lights along the side of a straight railway track. In the reference frame of the track the lights are spaced at regular intervals $L$, flash periodically with period $T$, and are synchronized so that all the lights flash simultaneously during a given period. The flashes constitute a spacetime lattice of events.

Now consider the situation in the reference frame of a train moving along the track at a constant velocity $\beta$. We would like to choose the values of $\beta$ and $\alpha \equiv L / T$ such that the lattice of events has the same properties in the train frame as it does in the track frame. ${ }^{14}$ Specifically, an observer in the rest frame of the train should see periodic flashes of light at positions $n L$ relative to some stationary reference position, where $n$ is an integer. The period of the flashes should be $T$, and the flashes during a given period should occur simultaneously. 15

We can determine the allowed values of $\beta$ and $\alpha$ by using the results of Sec. IV. From Eqs. (15), (18), and (28), it follows that

$$
A=B=T / 2, \quad C=-D=L / 2 .
$$

If we substitute Eq. (29) into Eq. (25), we find that

$$
\alpha \equiv L / T=(\lambda-a) / b=-\left(\lambda^{-1}-a\right) / b .
$$

We can use Eq. (30) to solve for $\gamma$ and $\alpha$ in terms of $a$ and $b$ :

$$
\gamma=a, \quad \alpha=\sqrt{a^{2}-1} / b .
$$

From Eqs. (23), (24), and (31), it follows that

$$
c=\left(a^{2}-1\right) / b, \quad d=a .
$$

Thus, once we specify the pair of integers $(a, b)$, the parameters $\alpha$ and $\gamma$ are uniquely determined by Eq. (31), and the parameters $c$ and $d$ are uniquely determined by Eq. (32).

Not all pairs of integers $(a, b)$ yield viable lattices. Because $\gamma \geq 1$ and $\alpha>0$, Eq. (31) implies that $a>1$ and $b$ $>0$. Because $c$ must be an integer, Eq. (32) implies that $b$ must divide $a^{2}-1$. It is straightforward to check that these three conditions are sufficient: the pair of integers $(a, b)$ will always yield a viable lattice provided that $a>1, b>0$, and $b$ divides $a^{2}-1$.

Note that if $(a, b)$ yields a viable lattice, then so does $\left(a, b^{\prime}\right)$, where $b^{\prime}=\left(a^{2}-1\right) / b$. If we let $\{\alpha, \gamma, c, d\}$ denote the parameters corresponding to lattice $(a, b)$, and $\left\{\alpha^{\prime}, \gamma^{\prime}, c^{\prime}, d^{\prime}\right\}$ denote the parameters corresponding to lattice $\left(a, b^{\prime}\right)$, then from Eqs. (31) and (32) it follows that the two sets of parameters are related by

$$
\alpha^{\prime}=1 / \alpha, \quad \gamma^{\prime}=\gamma, \quad c^{\prime}=b, \quad d^{\prime}=d .
$$

If $a>b$, then $\alpha>1$, and if $a<b$, then $\alpha<1$.

Let us now consider some specific examples. We first note that the three viability conditions are always satisfied for $a$ $>1$ and $b=1$. The lattice parameters in this case are

$$
\gamma=a, \quad \alpha=\sqrt{a^{2}-1}, \quad c=a^{2}-1, \quad d=a .
$$

From Eq. (31), it follows that for a lattice with basis vectors given by Eq. (28) the smallest possible value of $\gamma$ is $\gamma=2$, corresponding to $\beta=\sqrt{3} / 2$. A lattice with $\gamma=2$ is described by Eq. (34) with $a=2$. The remaining lattice parameters are $b=1, c=3, d=2$, and $\alpha=\sqrt{3}$.

Several other viable lattices are described in Table I, which gives a complete list of viable lattices such that 1 $<b<a<7$. The parameters for lattices with $a<7$ and $b$ $>a$ can be obtained from Table I via Eq. (33).

We can interpret these results by returning to our example of an array of flashing lights. Let us first interpret the solution with $a>1$ and $b=1$ that is described by Eq. (34). We see that if the ratio of the light spacing $L$ to the flash period $T$ is $\alpha=L / T=\sqrt{a^{2}-1}$, then the spacetime lattice of flashes will appear the same in the reference frame of the track and in the reference frame of a train moving with a gamma factor of 
Table I. List of integer pairs $(a, b)$ that yield viable lattices and the corresponding values of the parameters $\alpha, \gamma, c$, and $d$.

\begin{tabular}{lcccc}
\hline \hline$(a, b)$ & $\alpha$ & $\gamma$ & $c$ & $d$ \\
\hline$(3,2)$ & $\sqrt{2}$ & 3 & 4 & 3 \\
$(4,3)$ & $\sqrt{5 / 3}$ & 4 & 5 & 4 \\
$(5,2)$ & $\sqrt{6}$ & 5 & 12 & 5 \\
$(5,3)$ & $\sqrt{4 / 3}$ & 5 & 8 & 5 \\
$(5,4)$ & $\sqrt{3 / 2}$ & 5 & 6 & 5 \\
$(6,5)$ & $\sqrt{7 / 5}$ & 6 & 7 & 6 \\
\hline \hline
\end{tabular}

$\gamma=a$. Table I gives some additional values of $\alpha$ and $\gamma$ for which the lattice of flashes appears the same in both reference frames.

\section{TRIANGULAR LATTICES}

Let us now consider a second class of lattices whose basis vectors $e_{1}^{\mu}$ and $e_{2}^{\mu}$ have the form

$$
e_{1}^{\mu}=(T, L), \quad e_{2}^{\mu}=(T,-L)
$$

for some constants $T$ and $L$. A lattice of this form is shown in Fig. 2.

We can give an example of a set of events that constitute a lattice of this form by considering again an array of periodically flashing lights along the side of a railway track. Let us number the lights relative to a reference light at $x=0$. For a lattice of the form described by Eq. (35), flashes from evennumbered lights alternate with flashes from odd-numbered lights, where each set of lights flashes periodically with period $2 T$ and the delay between flashes from the two sets is $T$. As before, we consider the situation in the reference frame of a train moving along the track at a constant velocity $\beta$. We would like to determine the allowed values of $\beta$ and $\alpha$ $=L / T$ such that the spacetime lattice of flashes has the same properties in the train frame as it does in the track frame.

The treatment of this class of lattices closely parallels the treatment for the class of lattices considered in Sec. V, so we will omit the derivations and just state the results. As before, we find that the pair of integers $(a, b)$ uniquely determines the parameters $\alpha \equiv L / T, \gamma, c$, and $d$. The parameters $\gamma$ and $\alpha$ are given by

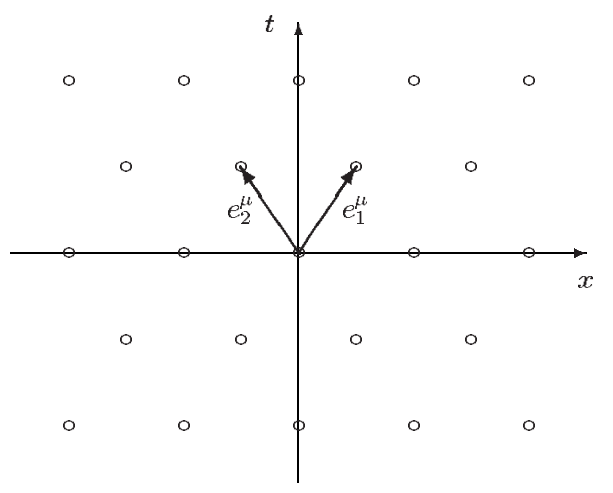

Fig. 2. Spacetime lattice generated by the basis vectors $e_{1}^{\mu}$ and $e_{2}^{\mu}$ defined in Eq. (35). The circles indicate events $R_{m, n}^{\mu}$ that belong to the lattice.
Table II. List of integer pairs $(a, b)$ that yield viable lattices and the corresponding values of the parameters $\alpha, \gamma, c$, and $d$.

\begin{tabular}{lcccc}
\hline \hline$(a, b)$ & $\alpha$ & $\gamma$ & $c$ & $d$ \\
\hline$(8,3)$ & $\sqrt{5}$ & $7 / 2$ & -3 & -1 \\
$(8,5)$ & $\sqrt{21}$ & $5 / 2$ & -5 & -3 \\
$(12,5)$ & $\sqrt{6}$ & 5 & -5 & -2 \\
$(12,7)$ & $\sqrt{15}$ & 4 & -7 & -4 \\
$(15,4)$ & $\sqrt{3}$ & 7 & -4 & -1 \\
$(15,11)$ & $\sqrt{45}$ & $7 / 2$ & -11 & -8 \\
$(16,7)$ & $\sqrt{33 / 5}$ & $13 / 2$ & -7 & -3 \\
$(16,9)$ & $\sqrt{13}$ & $11 / 2$ & -9 & -5 \\
\hline
\end{tabular}

$$
\gamma=\left(1+a^{2}-b^{2}\right) / 2 a, \quad \alpha=\sqrt{\frac{(a+b)^{2}-1}{(a-b)^{2}-1}},
$$

and the parameters $c$ and $d$ are given by

$$
c=-b, \quad d=\left(1-b^{2}\right) / a .
$$

One can show that the pair of integers $(a, b)$ yields a viable lattice if and only if $a>0,0<|b|<a-1$, and $a$ divides $b^{2}$ -1 .

If the pair of integers $(a, b)$ yields a viable lattice, then so does the pair $(a,-b)$. If we let $\{\alpha, \gamma, c, d\}$ denote the parameters corresponding to lattice $(a, b)$, and $\left\{\alpha^{\prime}, \gamma^{\prime}, c^{\prime}, d^{\prime}\right\}$ denote the parameters corresponding to lattice $(a,-b)$, then the two sets of parameters are related by Eq. (33). If $b>0$, then $\alpha>1$, so the basis vectors $e_{1}^{\mu}$ and $e_{2}^{\mu}$ are spacelike; if $b<0$, then $\alpha<1$, so the basis vectors are timelike.

Let us now consider some specific lattices. We first note that the three viability conditions are always satisfied for $a$ $>2$ and $b=1$. The lattice parameters in this case are

$$
\alpha=\sqrt{\frac{a+2}{a-2}}, \quad \gamma=a / 2, \quad c=-1, \quad d=0 .
$$

In Sec. IV we showed that $\gamma$ must be an integer or halfinteger, so the smallest possible nontrivial value of $\gamma$ is $\gamma$ $=3 / 2$, corresponding to $\beta=\sqrt{5} / 3$. A lattice with $\gamma=3 / 2$ is described by Eq. (38) with $a=3$. The remaining lattice parameters are $b=1, c=-1, d=0$, and $\alpha=\sqrt{3}$.

Several other viable lattices are described in Table II, which gives a complete list of viable lattices $(a, b)$ such that $a<20$ and $b>1$. The parameters for lattices with $a<20$ and $b<-1$ can be obtained from Table II using Eq. (33).

We can interpret these results by returning to our example of an array of flashing lights. First consider the solution with $a>2$ and $b=1$ that is described by Eq. (38). We see that if the ratio of the light spacing $L$ to the flash period $T$ is $\alpha$ $=L / T=\sqrt{(a+2) /(a-2)}$, then the spacetime lattice of flashes will appear the same in the reference frame of the track and in the reference frame of a train moving with a gamma factor of $\gamma=a / 2$. Table II gives several additional values of $\alpha$ and $\gamma$ for which the lattice appears the same in both frames. Recall that for lattices of the form considered in Sec. V, in which all the lights flash simultaneously during a given period, we found that $\gamma$ had to be an integer. In contrast, for lattices of the form considered here, in which flashes from evennumbered lights alternate with flashes from odd-numbered lights, we see that half-integer values of $\gamma$ are also allowed. 


\section{LATTICES IN HIGHER DIMENSIONS}

So far we have only considered periodic lattices in (1 +1 ) dimensions, and it is natural to ask how our results generalize to $(n+1)$ dimensions. One way to obtain a periodic lattice in $(n+1)$ dimensions is to embed multiple copies of a periodic lattice in $(1+1)$ dimensions. We will illustrate this technique by using it to obtain a periodic lattice in $(2+1)$ dimensions.

Start with a $(1+1)$-dimensional lattice whose basis vectors are given by Eq. (28). Define basis vectors $f_{1}^{\mu}, f_{2}^{\mu}$, and $f_{3}^{\mu}$ for a $(2+1)$-dimensional lattice by

$$
f_{1}^{\mu}=(T, 0,0), \quad f_{2}^{\mu}=(0, L, 0), \quad f_{3}^{\mu}=(0,0, L) .
$$

Consider a boost $B_{x}(\beta)$ of velocity $\beta$ along the $x$-axis. From Eqs. (13) and (39), it follows that

$$
\begin{aligned}
& B_{x}(\beta) f_{1}^{\mu}=a f_{1}^{\mu}+b f_{2}^{\mu}, \\
& B_{x}(\beta) f_{2}^{\mu}=c f_{1}^{\mu}+d f_{2}^{\mu}, \\
& B_{x}(\beta) f_{3}^{\mu}=f_{3}^{\mu} .
\end{aligned}
$$

Thus, the $(2+1)$-dimensional lattice that is generated by the basis vectors $f_{1}^{\mu}, f_{2}^{\mu}$, and $f_{3}^{\mu}$ is invariant under the boost $B_{x}(\beta)$. Similarly, one can show that the lattice is invariant under a boost $B_{y}(\beta)$ of velocity $\beta$ along the $y$-axis. By generalizing the arguments presented in Sec. III, we find that the lattice is invariant under translations through integer multiples of the basis vectors, under sequences of boosts along the $x$ - or $y$-axis with velocity $\pm \beta$, and under rotations through integer multiples of $\pi / 2$.

By generalizing this technique, we can obtain periodic lattices in $(n+1)$-dimensions. It seems likely that there are $(n+1)$-dimensional lattices with larger symmetry groups than can be obtained via this method. As far as I know, obtaining such lattices, and determining their associated symmetry groups, is an open problem.

\section{DISCUSSION}

We have considered the problem of finding a periodic lattice in Minkowski space that is left invariant by a discrete subgroup of the inhomogeneous Lorentz group. We have given a prescription for constructing such lattices and have described several examples. Our results illustrate basic concepts of spacetime symmetry and also show how ideas of crystal lattice structure can be applied in a novel context.

In addition to their pedagogical value, the periodic lattices we have discussed may be useful for simulating $(1+1)$-dimensional field theories on a computer. To simulate a field theory on a computer, we must discretize the theory to render the number of degrees of freedom finite. By discretizing a field theory using one of the periodic lattices described here, the resulting discrete theory would have a symmetry group that is a discrete subgroup of the inhomogeneous Lorentz group. Such discrete field theories may also be interesting objects of study in their own right.

\section{ACKNOWLEDGMENTS}

The author would like to thank an anonymous reviewer for calling his attention to Ref. 3 and for making a number of helpful suggestions, including the treatment for the general solution presented in Sec. IV and the physical interpretation of spacetime lattices in terms of flashing lights.

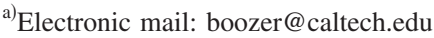

${ }^{1}$ An overview of crystal structure is given in N. W. Ashcroft and N. D. Mermin, Solid State Physics (Harcourt Brace, New York, 1976), pp. 6382, 111-128.

${ }^{2}$ See also A. Nussbaum, "The mystery of the fifteenth Bravais lattice," Am. J. Phys. 68 (10), 950-954 (2000).

${ }^{3}$ A. Janner and E. Ascher, "Bravais classes of two-dimensional relativistic lattices," Physica (Amsterdam) 45, 33-66 (1969).

${ }^{4}$ A derivation of the inhomogeneous Lorentz group for $(1+1)$-dimensional Minkowski space is given in K. A. Dunn, "Poincaré group as reflections in straight lines," Am. J. Phys. 49 (1), 52-55 (1981).

${ }^{5} \mathrm{~A}$ discussion of the homogeneous Lorenz group for $(n+1)$-dimensional Minkowski space is given in A. A. Ungar, "The abstract Lorentz transformation group," Am. J. Phys. 60 (9), 815-828 (1992).

${ }^{6}$ A discussion of the inhomogeneous Lorentz group for $(3+1)$-dimensional Minkowski space is given in S. Weinberg, The Quantum Theory of Fields I (Cambridge U. P., Cambridge, 1995), pp. 55-62.

${ }^{7}$ The inhomogeneous Lorentz group for $(3+1)$-dimensional Minkowski space is also discussed in R. Scurek, "Understanding the CPT group in particle physics: Standard and nonstandard representations," Am. J. Phys. 72 (5), 638-643 (2004); J. A. Morgan, "Spin and statistics in classical mechanics," ibid. 72 (11), 1408-1417 (2004).

${ }^{8}$ A. Schild, "Discrete space-time and integral Lorentz transformations," Can. J. Math. 1, 29-47 (1949).

${ }^{9}$ H. R. Coish, "Elementary particles in a finite world geometry," Phys. Rev. 114, 383-388 (1959).

${ }^{10}$ Here "field" is meant in the algebraic sense, as a commutative ring with multiplicative inverses, rather than in the geometric sense, as a quantity that depends on spacetime position.

${ }^{11}$ Technically, the group we have defined is the restricted inhomogeneous Lorentz group because it does not include space inversion or time reversal transformations. If these transformations are included, the resulting group is the general inhomogeneous Lorentz group, also known as the Poincaré group.

${ }^{12}$ A lattice of this form is known as a Bravais lattice; see Ref. 1, pp. 64-66.

${ }^{13}$ Here we have used the fact that a Lorentz transformation $\Lambda_{\nu}^{\mu}(\beta)$ has unit determinant and thus preserves spacetime areas.

${ }^{14}$ The analogous problem for Galilean transformations has a simple solution: any ratio $\alpha=L / T$ is allowed, and the corresponding velocity is $\beta$ $=\alpha$.

${ }^{15}$ Because of the relativity of simultaneity, flashes that are simultaneous in the train frame are not simultaneous in the track frame. 